\title{
Photochemically Active Dyes for Super-Resolution Microscopy
}

\author{
Zacharias Thiel and Pablo Rivera-Fuentes*
}

\begin{abstract}
The development of super-resolved optical microscopies has revolutionized the way we visualize cell biology. These techniques strongly rely on the use of photochemically active fluorophores that display changes in their photophysical properties upon irradiation with light. Many reversible and irreversible photochemical transformations have been explored for this purpose, and different imaging techniques require specific mechanisms of photoconversion. In this review, we provide an overview of the most common strategies used for the development of fluorophores for super-resolution microscopy and give specific examples of state-of-the-art fluorogenic probes. Furthermore, we discuss their main field of application and possible directions for future developments.
\end{abstract}

Keywords: Fluorescent probes · Live-cell imaging · Photoactivatable $\cdot$ Photoswitchable $\cdot$ Super-resolution microscopy
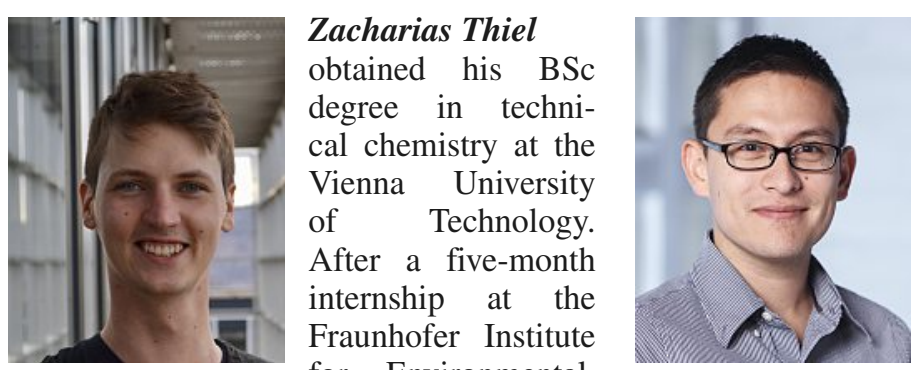

Pablo Rivera-Fuentes obtained his BSc degree in chemical engineering at the $\mathrm{Na}-$ tional Autonomous University of Mexico, and $\mathrm{MSc}$ and $\mathrm{PhD}$ degrees in chemistry from ETH Zurich. After postdoctoral Safety, and Energy Technology, he completed his MSc degree in chemistry at ETH Zurich. Since 2017, he has been PhD student at ETH Zurich, working on the development and implementation of singlemolecule fluorescent sensors of enzymatic activity. appointments at MIT and the University of Oxford, he joined the faculty at ETH Zurich as assistant professor in late 2015. His research focuses on the development of chemical tools to control and visualize biological processes in live cells with sub-organelle precision.

\section{Introduction}

Optical microscopy is an indispensable tool in life sciences because it facilitates the direct and non-invasive observation of living specimens. The advent of fluorescent proteins and biocompatible small-molecule dyes enabled the elucidation of molecular mechanisms employing fluorescence microscopy. Resolution, however, remains a major shortcoming of optical microscopy. Because of the wavelike nature of light, the signals of single emitters are detected as blurred spots. The dimensions of these spots depend on the wavelength of light and the numerical aperture of the objective and limit the minimal distance between adjacent emitters that can be resolved to approximately $200 \mathrm{~nm}$, which is known as the diffraction limit. ${ }^{[1]}$ Small molecules, proteins, membranes, and most intracellular organelles are smaller than this and therefore cannot be resolved by conventional fluorescence microscopy. This challenge, however, has been recently overcome by the inception of super-resolved optical microscopy techniques.

One of these techniques is single molecule localization microscopy (SMLM), which include stochastic optical reconstruction microscopy (STORM), ${ }^{[2]}$ photoactivated localization microscopy (PALM), ${ }^{[3]}$ and related techniques. The prerequisite for SMLM is the use of probes that can transition between a non-fluorescent (dark) or a fluorescent (bright) state, usually by means of a photochemical reaction. One way to create a super-resolved image is to label the target structure with probes that are initially in their dark state. A small subset of probes is then stochastically converted into the bright state and the signals of these molecules are recorded. If the activated fluorophores are separated by a distance greater than the diffraction limit, their precise localizations can be determined by deconvolution of the signals recorded. The activated probes are then switched back to a dark state. The cycle of photoactivation, imaging, deconvolution, and deactivation is repeated multiple times. These individually deconvoluted frames can then be used to reconstruct a super-resolved image. The precision of the localization that can be obtained for each fluorophore depends on the number of photons that are collected while it resides in the bright state. The brightness (the product of the molar extinction coefficient and the fluorescence quantum yield) along with photostability are therefore crucial properties that need to be considered when designing probes for SMLM.

Another strategy to achieve resolutions beyond the diffraction limit is through spatial control of illumination of the 
sample, which is the basis of stimulated emission depletion (STED) ${ }^{[4]}$ and reversible saturable optical fluorescence transitions (RESOLFT). ${ }^{[5]}$ In these methods, the specimen is scanned by two laser beams; an excitation beam excites the fluorophores and a superimposed deactivation beam converts the fluorophores into a dark state (RESOLFT) or forces them back into the ground state by stimulated emission (STED). The deactivation beam is optically modulated to adopt a doughnut shape with a minimum in the center. Only the molecules at the center of this beam are not depleted and remain in a bright state. The combination of the two beams results in an effective excitation spot that is smaller than the diffraction limit.

Alternatively, structured illumination microscopy (SIM) overcomes the diffraction limit by illuminating the sample with superimposed striped patterns of light. With linear SIM,[6] a twofold improvement of resolution is obtained and conventional fluorophores can be used. Even higher resolutions can be obtained when the concept is extended into non-linear regimes with probes that enable the transition between a bright and a dark state. ${ }^{[7]}$ For both RESOLFT and non-linear SIM, the photoactive fluorophores must undergo multiple switching cycles between the dark and the bright state. High fatigue resistance (the number of reversible transitions that a probe can undergo before photobleaching) is therefore a key requirement for longterm imaging using these techniques.

The success of SMLM, RESOLFT, and non-linear SIM depends on the photophysical properties of the fluorophores that are used. The fundamental requirement for these probes is their ability to transition between a dark and a bright state, but additional characteristics make certain fluorophores advantageous for one technique over another. In this review, we define these characteristics and provide an overview of currently available dyes and their applications. We conclude by pointing into possible future directions in the development and application of this class of probes.

\subsection{Definitions}

Photochemically active fluorophores can be categorized based on the processes that lead to the formation of the bright and dark states and the reversibility of these processes. Irreversibly switchable probes are permanently converted from one state to another by irradiation with light. The transition can either be from a non-fluorescent to a fluorescent state (photoactivatable probes) or between two fluorescent states with different emission wavelengths (photoconvertible probes). Returning the probes back into a dark state after read- out can only be obtained by permanent photobleaching. Reversibly switchable probes (photoswitchable) undergo a reversible light-induced transition from a non-fluorescent to a fluorescent state. This photoreaction is reversible and the fluorophores can be transitioned back into the dark state. This conversion occurs either spontaneously as a thermal process or is photochemically induced. Spontaneously blinking fluorophores are a class of molecules that feature thermal interconversion between a dark and a fluorescent state. Both transitions occur via ground-state reactions, and therefore no additional irradiation is required.

\section{Fluorescent Proteins}

The use of fluorescent proteins in optical microscopy is well established and the development of novel, improved variants is still the subject of intensive research. ${ }^{[8]}$ Consequently, photochemically active fluorescent proteins have also emerged as important tools for super-resolution microscopy. Prominent representatives are the photoactivatable variant of the green fluorescent protein PA-GFP, ${ }^{[9]}$ the photoconvertible proteins Kaede ${ }^{[10]}$ and EosFP, ${ }^{[11]}$ and the photoswitchable protein Dronpa.[12] Some of these genetically encoded fluorescent markers were used for the develop-

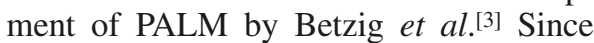
then, a broad selection of photochemically active proteins that are optimized for superresolution microscopy has been developed. Fluorescent proteins are expressed by the specimen itself which circumvents tedious labeling protocols. Furthermore, they can be used as fusion proteins to targets of interest, providing excellent labeling specificity. The benefits of intracellular fluorophore production are, however, attenuated by several disadvantages. For instance, expression and maturation can be slow, sensitive to the chemical environment, and feature strong variation in efficiency among different cell types. ${ }^{[13]}$ Moreover, fluorescent proteins generally photobleach faster than small-molecule fluorophores. ${ }^{[13]}$ Considering that super-resolved techniques usually require irradiation with light of high intensity, fluorescent proteins are useful only for short-term experiments because of their rapid photobleaching.

\section{Small-molecule Dyes}

Small-molecule fluorophores usually display higher fluorescence quantum yields, brightness, and photostability than fluorescent proteins. ${ }^{[14]}$ The photophysical properties of small-molecule dyes can also be tuned by rational design, which has led to the development of fluorophores of various emission wavelengths, excited-state lifetimes, Stokes shifts, etc. Importantly, a number of labeling strategies has been developed to tag macromolecules with small-molecule dyes. Examples include the self-labeling proteins SNAP-tag, ${ }^{115}$ CLIP-tag, ${ }^{[16]}$ and HaloTag, ${ }^{[17]}$ labeling via various engineered ligases and transferases, ${ }^{[18]}$ specific binding of small molecules to short peptide-tags, ${ }^{[19,20]}$ and bioconjugation using a broad variety of bioorthogonal reactions. ${ }^{[21]}$

Because of these advantages, we focus on small-molecule photochemically active dyes in this review. The development and applications of fluorescent proteins in super-resolved optical microscopy has been reviewed elsewhere. ${ }^{[22-24]}$

\subsection{Photoactivatable Probes}

Irreversibly photoactivatable probes are normally fluorophores that have been modified with photolabile moieties that block them in a non-fluorescent state. Irradiation of the initially non-emissive molecules with light of certain wavelengths induces a photochemical reaction that liberates a fluorophore (photoactivation). Photoactivatable probes are suitable for localization based super-resolution microscopy techniques such as PALM and STORM.

\subsection{1 o-Nitrobenzylalcohol Derivatives}

$o$-Nitrobenzylalcohol (NB) derivatives are among the most commonly used photocleavable protecting groups. Fluorophores can be modified with NB, which quenches their fluorescence. Irradiation with UV light triggers the conversion to $o$-nitrosobenzaldehyde and liberates the fluorophore. ${ }^{[25]}$ Fluorophores modified with NB have been used as photoactivatable fluorogenic substrates in microscopy studies long before the development of SMLM. A photoactivatable, NB-protected fluorescein 1a, which releases $\mathbf{1 b}$ (Scheme 1), was used to label microtubules inside cells and enabled the observation of spindle dynamics during mitosis. ${ }^{[26]}$ The closely related 6-nitroveratryloxycarbonyl group (NVOC) is more commonly used to mask probes for super-resolution microscopy. The two methoxy groups shift the absorption to longer wavelengths to allow for efficient photoactivation at $405 \mathrm{~nm}$, increasing the compatibility of the probes with standard fluorescence microscopes. ${ }^{[27,28]}$ Several fluorophores containing fluorescein and rhodamine derivatives, ${ }^{[29-31]}$ silicon rhodamines, ${ }^{[32]}$ and a quinonecyanine-derivative ${ }^{[33]}$ have been modified with NB and NVOC and successfully applied for SMLM experiments. The popularity of this photoactivatable group arises from its relatively easy preparation and the 
compatibility with a broad range of fluorophores. The main disadvantage of these photoactivatable groups are the byproducts that are formed during the photoreaction, which are highly reactive and toxic species, and therefore not optimal for livecell imaging. ${ }^{[34]}$

\subsubsection{Reductive Conversion of Conventional Fluorophores}

Several carbocyanine and rhodamine derivatives can be locked in a non-fluorescent state by reduction with $\mathrm{NaBH}_{4}$. For instance, the non-emissive hydrocyanine $\mathbf{3 a}$ is obtained from $\mathbf{3 b}$ (Scheme 1). Upon

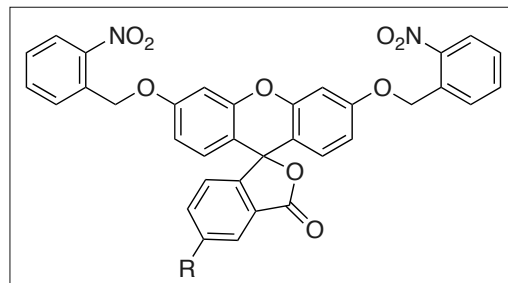

$1 \mathbf{a}$

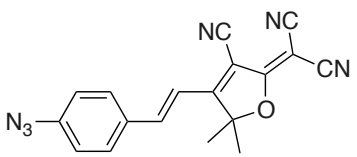

$2 a$

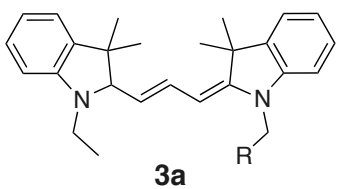

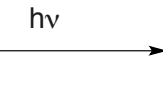

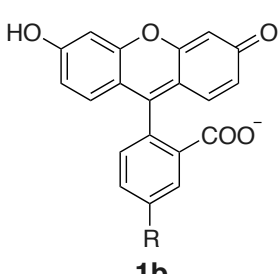

$1 b$

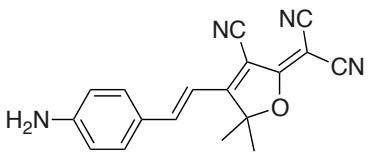

$2 b$

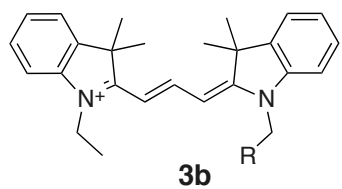

Scheme 1. Photoactivatable compounds that undergo irreversible transition from a non-fluorescent state to a fluorescent state upon irradiation.

\subsubsection{Azido Push-Pull Fluorophores}

Azide-substituted fluorophores were first used in SMLM by Moerner and coworkers. ${ }^{[35]}$ The electron-withdrawing azide moiety in $\mathbf{2 a}$ (Scheme 1) disrupts the push-pull system of the parent chromophore, quenching its fluorescence. Irradiation with blue light $(407 \mathrm{~nm})$ induces photoelimination of $\mathrm{N}_{2}$ and formation of a nitrene intermediate, which can further rearrange to an amine compound $\mathbf{2 b}$. The electron-donating character of the amine is sufficient to restore fluorescence. In a follow-up study, these probes were used as substrate-conjugates for self-labeling enzymes to show the spatial distribution of various targets in live bacteria. ${ }^{[36]} \mathrm{A}$ remarkable aspect of these probes is the high sensitivity of the photoactivatable group. The imaging experiments were performed without the aid of a blue activation laser, since ambient light (e.g. from a nearby computer screen) already provided enough photoactivation. ${ }^{[36]}$ The applicability of these dyes without activation lasers might make them attractive for imaging applications where phototoxicity might pose problems, but the extreme sensitivity of this functional group to light also makes it difficult to handle and therefore impractical. irradiation with blue light, the dyes return to their original fluorescent form. ${ }^{[37]}$ This is an elegant strategy that allows for facile modification of conventional fluorophores to make them applicable for super-resolution microscopy. A palette of commercially available fluorophores was assessed for their potential use as reductively caged probes, which offered a broad selection of possible emission wavelengths. ${ }^{[38]}$ The versatility of this approach was demonstrated by performing dual-color SMLM of synaptic vesicles using standard rhodamine-derivatives. This method, however, is not generally compatible with live-cell imaging because of the labeling protocols. The reduction of the fluorophores is usually performed on fixed cells after antibody labeling of the samples and harsh buffer systems are required for imaging. Furthermore, oxidation of the probes by endogenous reactive oxygen species might lead to uncontrolled activation. ${ }^{[39]}$

\subsubsection{Rhodamine-NN}

Belov et al. ${ }^{[40]}$ reported a convenient way to prepare photoactivatable xanthene dyes by a relatively simple chemical modification. In these rhodamine-NN dyes, the lactone of common rhodamines is exchanged by an $\alpha$-diazoindanone, which locks it in the non-fluorescent spirocyclic form. Irradiation with blue light and subsequent extrusion of $\mathrm{N}_{2}$ generates a carbene intermediate that undergoes Wolff rearrangement to give a highly fluorescent rhodamine analog. Employing this principle, a variety of rhodamine, carbopyronine, and silicon-rhodamine analogs were prepared, expanding the available emission spectra to the far red. ${ }^{41-43]}$ These fluorophores are especially well suited for live-cell imaging applications because the photoremovable group is small and non-polar and therefore does not interfere with cell-permeability. Furthermore, photolysis releases $\mathrm{N}_{2}$ as a non-toxic, non-absorbing byproduct. The utility of this type of photoactivatable compounds for live-cell super-resolution imaging was demonstrated by probes that were modified with substrates for self-labeling enzymes HaloTag and SNAP-tag.[43] These probes were used to label a variety of proteins and analyze their dynamic behavior with a combination of single-molecule tracking and PALM (sptPALM). These experiments enabled the observation of the trajectories of individual enzymes and the quantification of their diffusion coefficients.

Recently, our group employed the rhodamine-NN scaffold to develop the first photoactivatable probe for imaging enzymatic activity with super-resolution. ${ }^{44]}$ This probe relies on the divergent pathways of the Wolff rearrangement depending on the electron density of the xanthene system. Irradiation of diazoindanone $\mathbf{4 a}$ (Scheme 2), which bears an electronwithdrawing acetyl group yields mainly the non-fluorescent product $\mathbf{4 b}$. If, however, human carboxylesterases hydrolyze the acetyl group prior to irradiation, the highly fluorescent product $\mathbf{4 c}$ is obtained. The dependence of the fluorescent signal on enzymatic pre-activation enables the acquisition of precise spatial and temporal information about the distribution of active enzymes in live, genetically unmodified cells. The mechanism that was introduced in this proof-of-principle study has great potential for the future development of reporters and sensors for various analytes suitable for super-resolution microscopy.

\subsection{Photoswitchable Probes}

Photoswitchable probes can be reversibly transitioned between a dark and a bright state. Various mechanisms exist that are exploited for modulating the fluorescence of fluorophores. Reversibly switchable probes are suitable for SMLM as well as RESOLFT and non-linear SIM.

\subsubsection{Redox Switching of Cyanine Dyes}

A possible switching mechanism is the reversible addition of nucleophiles to car- 


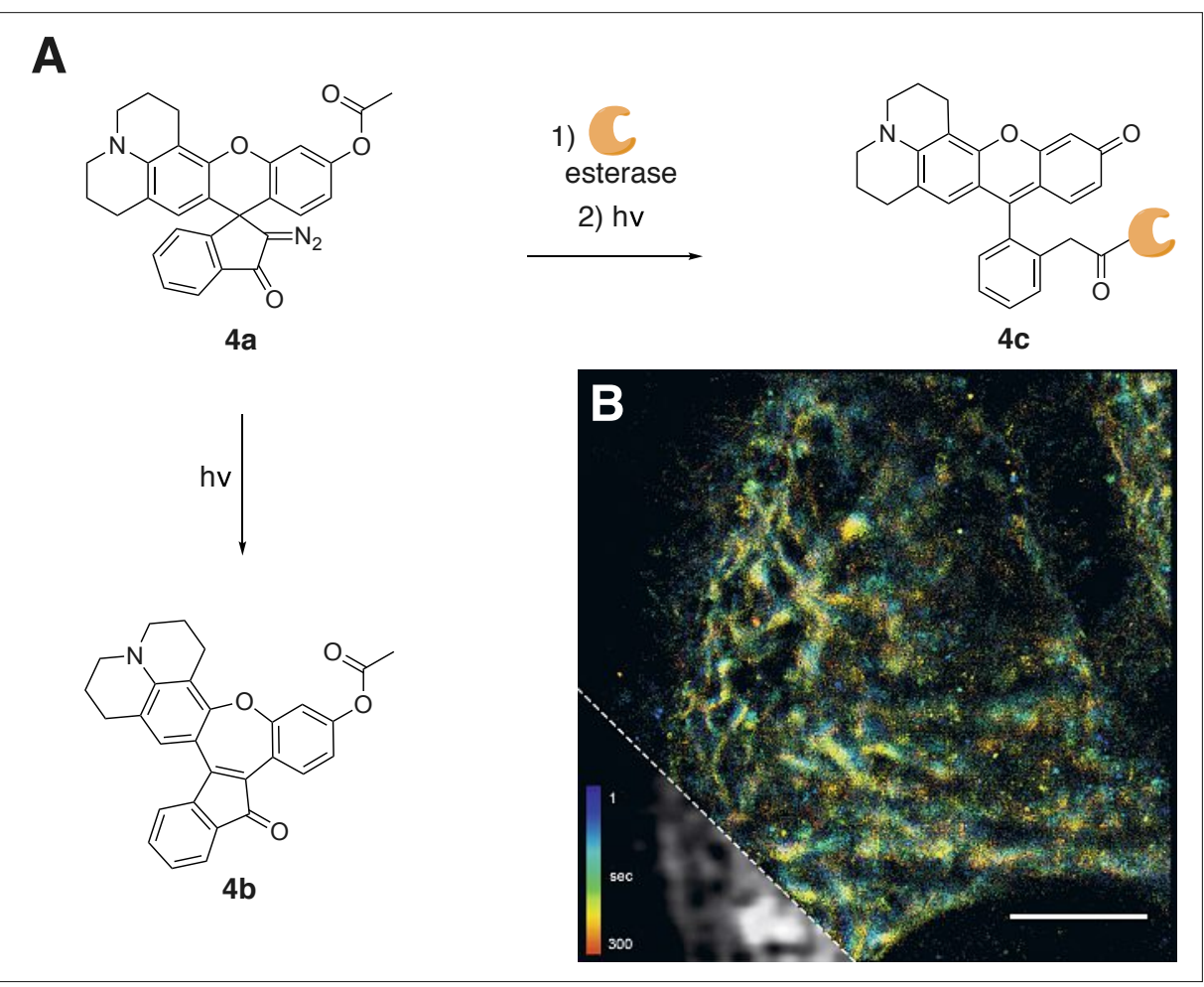

Scheme 2. (A) Mechanism for sensing esterase activity using the photoactivatable probe 4a. (B) Super-resolved image of esterase activity using $\mathbf{4 a}$ in a live HeLa cell. The signals are color coded according to their time of appearance. The diffraction-limited image is displayed in the lower left corner in black and white. Scale bar $=5 \mu \mathrm{m}$.

bocyanine dyes. Depending on the nucleophile, this process occurs under irradiation with red light(thiols) or spontaneously in the absence of light (trialkylphosphines). ${ }^{[45,46]}$ In either case, the nucleophile covalently adds to the polymethine bridge of the cya- nine dye 5a, breaking its conjugation and inducing a dramatic blue shift in absorption (Scheme 3). Irradiation of $\mathbf{5 b}$ with ultraviolet or blue light induces rapid dissociation of the adduct, restoring fluorescence. The efficiency of restoration at longer wavelengths can be substantially increased by using reporter-activator pairs. ${ }^{[47]}$ Excitation of a $\mathrm{Cy} 3$ activator leads to the restoration of a quenched Cy5 reporter dye in close proximity via resonance energy transfer. These carbocyanine-based activator-reporter pairs were already used in very early reports of SMLM experiments achieving nanometer resolution, ${ }^{[48,49]}$ as well as for RESOLFT nanoscopy of fixed cells in presence of KI. ${ }^{[50]}$ Even though excellent results were obtained in fixed samples, application of this strategy in live-cells is impaired by the requirement of overly reducing, toxic imaging buffers.

\subsubsection{Rhodamine Spiroamides}

A different switching mechanism that does not require the addition of external nucleophiles is exploited in photochromic rhodamine spiroamides. Upon irradiation with UV light, the $\mathrm{C}-\mathrm{N}$ bond of the spirolactam breaks, leading to the transient formation of a fluorescent zwitterionic species with a lifetime that is long enough to allow for the collection of sufficient photons for precise localization. ${ }^{[51,52]}$ Photoactivation of the rhodamine spiroamides that were

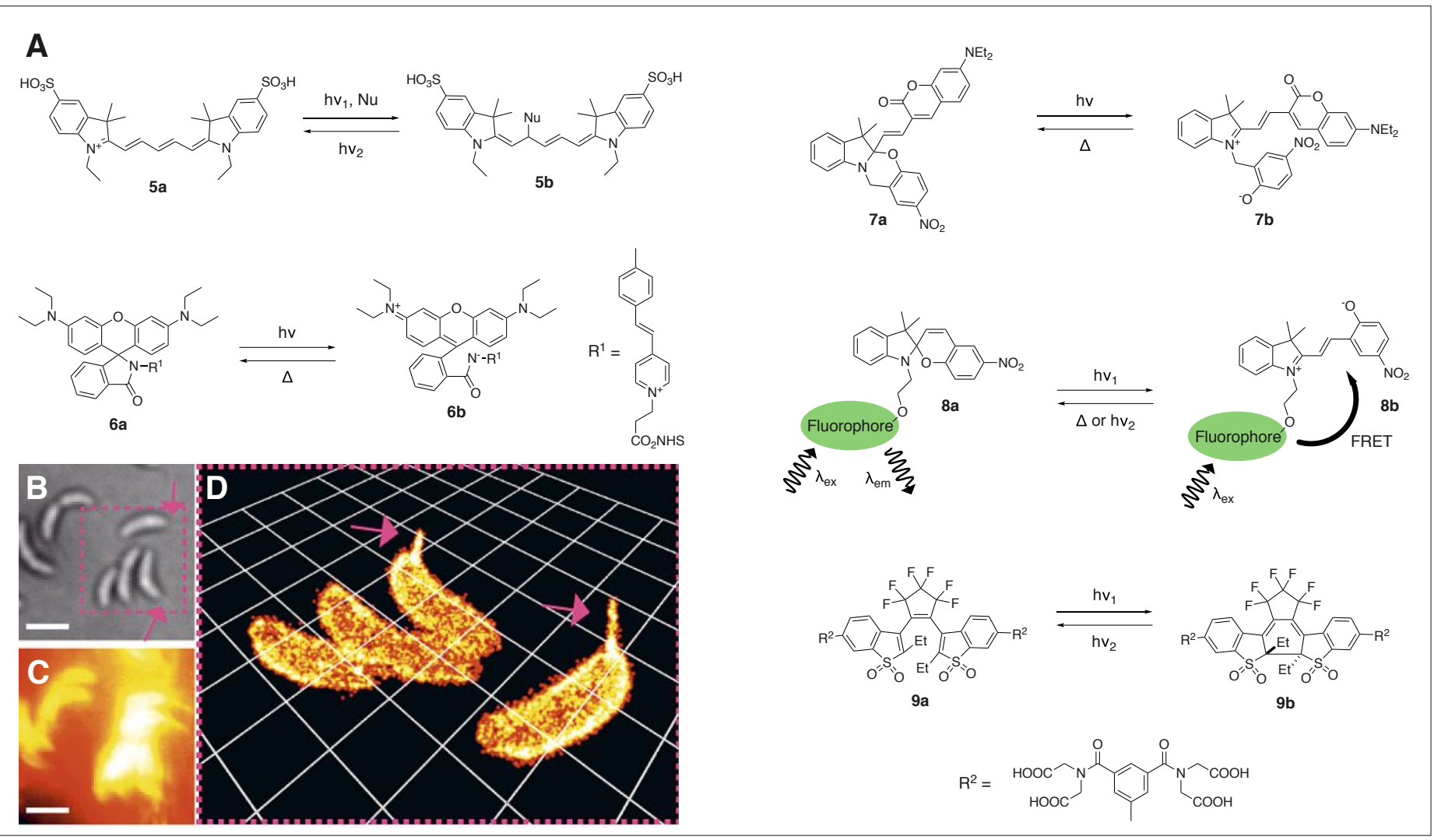

Scheme 3. (A) Photoswitchable compounds that undergo reversible transitions between fluorescent and non-fluorescent states. Nu = nucleophile. $(B, C$, and $D)$ Brightfield image, diffraction limited fluorescence image, and 3D SMLM image of bacteria using compound 6a, adapted from ref. [53] with permission. Scale bars $=1 \mu \mathrm{m}$. 
first reported, however, required irradiation with toxic UV light. Optimization of the substituent on the spiroamide nitrogen allowed photoactivation with longer wavelengths, improving the applicability of $\mathbf{6 a}$ for live-cell imaging (Scheme 3).[53] Consequently, the performance of $\mathbf{6 a}$, which transiently forms $\mathbf{6 b}$ upon irradiation with $405 \mathrm{~nm}$, was demonstrated by imaging the surface of Gram-negative bacteria with 3D SMLM with resolutions below $20 \mathrm{~nm}$.

\subsubsection{Spirooxazines}

Spirooxazines are optically active spirocycles that undergo photoinduced, reversible ring-opening reactions. ${ }^{[54]}$ Based on this switching mechanism, Raymo and co-workers developed a series of fluorogenic probes that consist of different fluorophores connected to a $2 \mathrm{H}, 3 \mathrm{H}$-indole with a spirooxazine switching unit.[55] Upon irradiation, the spirooxazine of representative compound $\mathbf{7 a}$ temporarily opens, generating the zwitterionic isomer 7b with lifetimes in the sub-microsecond regime (Scheme 3). In this isomeric form, the indolium cation and the fluorophore are conjugated, resulting in bathochromically shifted absorption and emission spectra. These spirooxazine-based probes exhibit excellent fatigue resistance, allowing for hundreds of switching cycles. The performance for super-resolution imaging of biological samples, however, is somewhat constrained by the fact that solvent polarity and low $\mathrm{pH}$ influence the co-existence of both isomeric states thus generating unwanted background fluorescence. Furthermore, the short lifetime of the fluorescent state limits the number of photons that can be collected per switching cycle, eroding the localization precision. Nevertheless, bioconjugates of $7 \mathbf{a}$ were prepared and employed in SMLM experiments of immunolabeled samples. ${ }^{[56]}$

\subsubsection{Spiropyrans}

Another common strategy to modulate fluorescence is to use optically switchable probes that quench the emission of a covalently linked reporter dye via resonance energy transfer.[57] Benzospiropyrans are a frequently used scaffold for switchable quenchers that form such dyads with BODIPY ${ }^{[58]}$ or rhodamine ${ }^{[59]}$ derivatives as donor fluorophores. Irradiation of $\mathbf{8 a}$ with UV light induces the transformation from a colorless spiropyran (SP) to a merocyanine (MC) with strong absorption at longer wavelengths because of the extended $\pi$-system of structures such as $\mathbf{8 b}$ (Scheme 3). In the MC form, the probe quenches the fluorescence of the covalently attached fluorophore unit. The thermal stability of the MC is strongly dependent on the chemical environment with half-lives up to $10^{4} \mathrm{~s} .{ }^{[60,61]}$ The reverse reaction to the SP can, however, be accelerated by irradiation with visible light. ${ }^{[62]}$ Several modifications of SP-based derivatives have aimed at optimizing its performance in aqueous environments ${ }^{[60]}$ and switching at longer wavelengths.[63] Unfortunately, the application for super-resolution imaging is limited as a consequence of the poor fatigue resistance of the spiropyran switching unit. Recently, an optical switch was developed by Anderson and co-workers based on the structurally related spironaphtooxazine. ${ }^{[64]}$ These fluorophore-quencher dyads, consisting of a rhodamine connected to the spironaphtooxazine quencher, can be switched with $405 \mathrm{~nm}$ light featuring fast switching rates, high switching efficiency, and excellent fatigue resistance even in living specimens. These advantages render spironaphtooxazines superior to spiropyrans for live-cell imaging and make them promising candidates for applications in super-resolution microscopy techniques such as RESOLFT and non-linear SIM.

\subsubsection{Diarylethenes}

Diarylethenes, especially 1,2-dithienylethene derivatives, have been extensively studied as photochromic compounds. [65] When irradiated with UV light, these probes undergo a light-induced cyclization reaction to give a thermally stable, ring-closed isomer with bathochromically shifted absorption as a consequence of its extended $\pi$-conjugation. Irradiation with visible light induces the cycloreversion to the open isomer. The high fatigue resistance of some derivatives allows for multiple switching cycles with minimal photodegradation. ${ }^{[66]}$ Furthermore, these compounds feature fast switching kinetics, high photoconversion quantum yields, and large separation of the absorption bands, ${ }^{[67]}$ which makes them interesting as probes for RESOLFT and non-linear SIM. Diarylethenes have been implemented as photoswitchable units in different probe designs. The strong absorption of the closed isomer at long wavelengths allows for its application as quencher in fluorophore-quencher dyads. ${ }^{[67,68]}$ Alternatively, sulfone derivatives of diarylethenes feature intrinsic fluorescence in the closed form and can thus be used without a reporter dye. ${ }^{[69,70]}$ As a consequence of the hydrophobic character of diarylethenes, however, their photophysical properties have mostly been reported in organic solvents and are severely impaired in aqueous environments. ${ }^{[71,72]}$ One approach to overcome this obstacle was to incorporate the probe into a hydrophilic polymer, which was used for the development of a lysosome-specific probe used in SMLM experiments in live cells. ${ }^{[73]} \mathrm{A}$ different approach is the modification of the molecular structure of diarylethenes by incorporation of hydrophilic residues. Introduction of several carboxylic acids enabled sufficient solubility of compounds $\mathbf{9 a}$ and 9b (Scheme 3), maintaining the photophysical properties required for superresolution imaging. ${ }^{[71,72]}$ The good performance of these probes for both SMLM and RESOLFT was demonstrated on immunolabeled cells. These studies exemplify well how the relatively simple modification of already existing compounds can lead to the development of probes that are suitable for imaging applications.

\subsection{Spontaneously Blinking Probes}

Both photoactivatable and photoswitchable probes require the use of an activation laser that generates the fluorescent species in addition to the laser used for readout. In many cases, irradiation with $405 \mathrm{~nm}$ or even shorter wavelengths is required for efficient switching. This high-energy irradiation, however, is toxic to living organisms and can cause severe damage to the illuminated cells, especially when imaged for long periods. ${ }^{[74]}$ The first approaches that aimed at omitting the activation laser relied on the transformation of dyes into long-lived dark states by addition of oxygen scavengers. ${ }^{[75]}$ It is, however, desirable to develop spontaneously blinking fluorophores that do not require harmful activation lasers and do not depend on buffer systems that might disrupt the normal physiology of live specimens. This functionality requires precise fine-tuning of the fluorophores and thus only a few examples have been reported so far.

\subsubsection{Hydroxymethyl Silicon Rhodamine}

In a landmark study by Johnsson and co-workers, the ability of carboxy-substi-

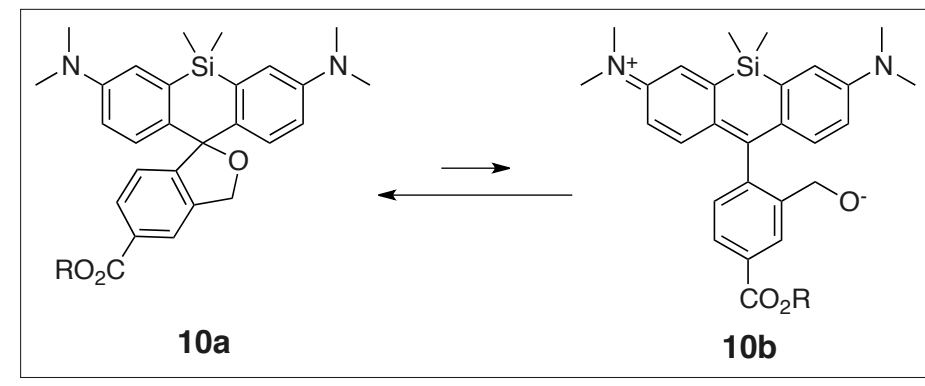

Scheme 4.

Mechanism of spontaneous blinking observed in fluorophore HMSiR 10a. 
tuted silicon rhodamine ( $\mathrm{SiR}$ ) to adopt its non-fluorescent spirocyclic form and transiently convert to the emissive isomer was noticed. ${ }^{[76]}$ On the basis of these blinking events, super-resolved SMLM experiments of histone H2B labeled by fusion to SNAPtag were performed in live and fixed cells.

By replacing the carboxylic acid of SiR with a hydroxymethyl moiety as intramolecular nucleophile, Urano and co-workers developed the spontaneously blinking fluorophore HMSiR 10a (Scheme 4). [77] The open and closed lifetimes of HMSiR were optimized for SMLM techniques but featured high sensitivity towards $\mathrm{pH}$ and the chemical environment. Schepartz, Toomre, and co-workers further developed these probes to obtain high-density, environment-sensitive (HIDE) membrane probes. ${ }^{[78]}$ The propensity of HMSiR to populate the non-fluorescent state in hydrophobic environments was thereby exploited. Placing HMSiR in such environments using membrane-targeting units reduced the fraction of HMSiR molecules in their emissive state to a minimum. Hence, very high labeling density could be used because the probability of overlapping signals from multiple probes simultaneously emitting was minimized. This labeling strategy allowed them to record unprecedented time-lapse imaging of live cells with high temporal and spatial resolution and slow apparent bleaching. Recently, another spontaneously blinking fluorophore was reported, expanding the range of available emission wavelengths to the green. ${ }^{[79]}$ The availability of this spectrally separated probe allowed for two-color SMLM imaging exclusively with blinking dyes.

\section{Concluding Remarks}

The advent of fluorescence-based superresolution microscopy techniques and the concomitant demand for photochemically active dyes entailed a tremendous amount of research dedicated to the development of new fluorogenic probes. Consequently, there is a plethora of available probes that can be chosen for the optimal design of imaging experiments. Nevertheless, there are still many potential features of fluorogenic probes that have not been addressed yet. Spontaneously blinking fluorophores, for example, bear great potential since they allow long-time image acquisition with little overall photobleaching and low phototoxicity. The probes available so far are, however, restricted to very specific chemical environments and perform well only for a limited range of cellular targets. Expanding their applicability to other environments is therefore highly desirable. With novel, fine-tuned spontaneously blinking fluorophores the acquisition of super-resolved images over extended periods under relatively gentle conditions can be possible. Such probes could prove to be powerful tools to gain insight into slow, biologically interesting processes. Furthermore, recent advances in the development of reversibly photoswitchable compounds lead to the introduction of probes with promising features for RESOLFT and non-linear SIM. A major drawback of the aforementioned probes is the fact that the wavelengths that are required for switching and readout are not decoupled. This feature has been achieved with a fluorescent, switchable protein and has proven to be very useful for imaging applications. ${ }^{[80]}$ More control over the duration of readout can be gained by decoupling the wavelengths that induce switching from the excitation wavelengths. Moreover, new super-resolution techniques, such as patterned activation non-linear SIM[81] and MINFLUX, ${ }^{82]}$ still require photoswitchable fluorophores to reach their potential. It is therefore unlikely that the need for photoactive probes will cease in the near future.

Ultimately, the development of fluorogenic sensors and reporters that are compatible with super-resolution microscopy would expand the scope of these techniques. These probes could reveal spatial and temporal organization of various analytes with unprecedented resolutions and prove extremely valuable for elucidating details of the spatiotemporal control of cellular processes. The development of new photochemically active probes, the optimization of already existing fluorophores, and their implementation into new and improved imaging techniques has the potential to revolutionize the way we visualize cell biology.

\section{Acknowledgements}

This work was supported by ETH Zurich. We thank Mr. Elias A. Halabi for critical reading of the manuscript.

Received: June 22, 2018

[1] E. Abbe, Arch. Mikrosk. Anat. 1873, 9, 413

[2] M. J. Rust, M. Bates, X. Zhuang, Nat. Methods 2006, 3, 793 .

[3] E. Betzig, G. H. Patterson, R. Sougrat, O. W. Lindwasser, S. Olenych, J. S. Bonifacino, M. W. Davidson, J. Lippincott-Schwartz, H. F. Hess, Science 2006, 313, 1642.

[4] S. W. Hell, J. Wichman, Opt. Lett. 1994, 19, 780.

[5] M. Hofmann, C. Eggeling, S. Jakobs, S. W. Hell, Proc. Natl. Acad. Sci. USA 2005, 102, 17565.

[6] M. G. L. Gustafsson, J. Microsc. 2000, 198, 82.

[7] E. H. Rego, L. Shao, J. J. Macklin, L. Winoto, G. A. Johansson, N. Kamps-Hughes, M. W. Davidson, M. G. L. Gustafsson, Proc. Natl. Acad. Sci. USA 2012, 109, E135.

[8] B. N. G. Giepmans, S. R. Adams, M. H. Ellisman, R. Y. Tsien, Science 2006, 312, 217.

[9] G. H. Patterson, J. Lippincott-Schwartz, Science 2002, 297, 1873.
[10] R. Ando, H. Hama, M. Yamamoto-Hino, H Mizuno, A. Miyawaki, Proc. Natl. Acad. Sci. USA 2002, 99, 12651.

[11] J. Wiedenmann, S. Ivanchenko, F. Oswald, F. Schmitt, C. Rocker, A. Salih, K.-D. Spindler, G. U. Nienhaus, Proc. Natl. Acad. Sci. USA 2004, $101,15905$.

[12] R. Ando, H. Mizuno, A. Miyawaki, Science 2004, 306, 1370 .

[13] P. J. Cranfill, B. R. Sell, M. A. Baird, J. R. Allen, Z. Lavagnino, H. M. de Gruiter, G.-J. Kremers, M. W. Davidson, A. Ustione, D. W. Piston, Nat. Methods 2016, 13, 557.

[14] S. J. Lord, H. D. Lee, W. E. Moerner, Anal. Chem. 2010, 82, 2192.

[15] A. Keppler, S. Gendreizig, T. Gronemeyer, H. Pick, H. Vogel, K. Johnsson, Nat. Biotechnol. 2003, 21, 86 .

[16] A. Gautier, A. Juillerat, C. Heinis, I. R. Corrêa M. Kindermann, F. Beaufils, K. Johnsson, Chem. Biol. 2008, 15, 128

[17] G. V. Los, L. P. Encell, M. G. McDougall, D. D Hartzell, N. Karassina, C. Zimprich, M. G. Wood, R. Learish, R. F. Ohane, M. Urh, D. Simpson, J. Mendez, K. Zimmermann, P. Otto, G. Vidugiris, J. Zhu, A. Darzins, D. H. Klaubert, R. F. Bulleit, K. V. Wood, ACS Chem. Biol. 2008, 3, 373.

[18] J. Lotze, U. Reinhardt, O. Seitz, A. G. BeckSickinger, Mol. BioSyst. 2016, 12, 1731.

[19] B. A. Griffin, S. R. Adams, R. Y. Tsien, Science 1998, 281, 269.

[20] T. L. Halo, J. Appelbaum, E. M. Hobert, D. M Balkin, A. Schepartz, J. Am. Chem. Soc. 2009, $131,438$.

[21] C. S. McKay, M. G. Finn, Chem. Biol. 2014, 21 , 1075.

[22] K. Nienhaus, G. Ulrich Nienhaus, Chem. Soc. Rev. 2014, 43, 1088

[23] D. M. Shcherbakova, P. Sengupta, J. LippincottSchwartz, V. V. Verkhusha, Annu. Rev. Biophys. 2014, 43, 303.

[24] S. N. Uno, D. K. Tiwari, M. Kamiya, Y. Arai, T. Nagai, Y. Urano, Microscopy 2015, 64, 263.

[25] J. A. Barltrop, P. J. Plant, P. Schofield, Chem. Commun. 1966, 822.

[26] T. J. Mitchison, J. Cell Biol. 1989, 109, 637.

[27] Y. R. Zhao, Q. Zheng, K. Dakin, K. Xu, M. L. Martinez, W. H. Li, J. Am. Chem. Soc 2004, $126,4653$.

[28] T. Kobayashi, Y. Urano, M. Kamiya, T. Ueno, H. Kojima, T. Nagano, J. Am. Chem. Soc. 2007, $129,6696$.

[29] S. Hauke, A. von Appen, T. Quidwai, J. Ries, R. Wombacher, Chem. Sci. 2017, 8, 559 .

[30] S. Banala, D. Maurel, S. Manley, K. Johnsson, ACS Chem. Biol. 2012, 7, 289.

[31] L. M. Wysocki, J. B. Grimm, A. N. Tkachuk, T. a. Brown, E. Betzig, L. D. Lavis, Angew. Chem. Int. Ed. 2011, 50, 11206.

[32] J. B. Grimm, T. Klein, B. G. Kopek, G. Shtengel, H. F. Hess, M. Sauer, L. D. Lavis, Angew. Chem. Int. Ed. 2016, 55, 1723.

[33] D.-P. Klötzner, K. Klehs, M. Heilemann, A Heckel, Chem. Commun. 2017, 53, 9874.

[34] A. P. Pelliccioli, J. Wirz, Photochem. Photobiol. Sci. 2002, 1, 441

[35] S. J. Lord, N. R. Conley, H. D. Lee, R. Samuel, N. Liu, R. J. Twieg, W. E. Moerner, J. Am. Chem. Soc. 2008, 130, 9204.

[36] H. L. D. Lee, S. J. Lord, S. Iwanaga, K. Zhan, H Xie, J. C. Williams, H. Wang, G. R. Bowman, E. D. Goley, L. Shapiro, et al., J. Am. Chem. Soc. 2010, 132, 15099.

[37] J. C. Vaughan, S. Jia, X. Zhuang, Nat. Methods 2012, 9, 1181.

[38] M. Lehmann, B. Gottschalk, D. Puchkov, P. Schmieder, S. Schwagerus, C. P. R. Hackenberger, V. Haucke, J. Schmoranzer, Angew. Chem. Int. Ed. 2015, 54, 13230

[39] K. Kundu, S. F. Knight, N. Willett, S. Lee, W. R. Taylor, N. Murthy, Angew. Chem. Int. Ed. 2009, 48, 299. 
[40] V. N. Belov, C. A. Wurm, V. P. Boyarskiy, S. Jakobs, S. W. Hell, Angew. Chem. Int. Ed. 2010 49,3520 .

[41] V. N. Belov, G. Y. Mitronova, M. L. Bossi, V. P. Boyarskiy, E. Hebisch, C. Geisler, K. Kolmakov, C. A. Wurm, K. I. Willig, S. W. Hell, Chem. Eur. J. 2014, 20, 13162.

[42] K. Kolmakov, C. Wurm, M. V Sednev, M. L. Bossi, V. N. Belov, S. W. Hell, Photochem. Photobiol. Sci. 2012, 11, 522.

[43] J. B. Grimm, B. P. English, H. Choi, A. K. Muthusamy, B. P. Mehl, P. Dong, T. A. Brown, J. Lippincott-Schwartz, Z. Liu, T. Lionnet, L. D. Lavis, Nat. Methods 2016, 13, 985.

[44] E. A. Halabi, Z. Thiel, N. Trapp, D. Pinotsi, P. Rivera-Fuentes, J. Am. Chem. Soc. 2017, 139, 13200.

[45] G. T. Dempsey, M. Bates, W. E. Kowtoniuk, D. R. Liu, R. Y. Tsien, X. Zhuang, J. Am. Chem. Soc. 2009, 131, 18192.

[46] J. C. Vaughan, G. T. Dempsey, E. Sun, X. Zhuang, J. Am. Chem. Soc. 2013, 135, 1197.

[47] M. Bates, T. R. Blosser, X. Zhuang, Phys. Rev. Lett. 2005, 94, 108101.

[48] M. Bates, B. Huang, G. T. Dempsey, X. Zhuang, Science 2007, 317, 1749.

[49] B. Huang, S. A. Jones, B. Brandenburg, X. Zhuang, Nat. Methods 2008, 5, 1047.

[50] J. Kwon, J. Hwang, J. Park, G. R. Han, K. Y. Han, S. K. Kim, Sci. Rep. 2015, 5, 17804.

[51] J. Fölling, V. Belov, R. Kunetsky, R. Medda, A. Schönle, A. Egner, C. Eggeling, M. Bossi, S. W. Hell, Angew. Chem. Int. Ed. 2007, 46, 6266.

[52] V. N. Belov, M. L. Bossi, J. Fölling, V. P. Boyarskiy, S. W. Hell, Chem. Eur. J. 2009, 15, 10762.

[53] M. K. Lee, P. Rai, J. Williams, R. J. Twieg, W. E. Moerner, J. Am. Chem. Soc. 2014, 136, 14003.

[54] M. Tomasulo, S. Sortino, A. J. P. White, S. Chimiche, A. Doria, J. Org. Chem. 2005, 70, 8180.

[55] E. Deniz, M. Tomasulo, J. Cusido, I. Yildiz, M. Petriella, M. L. Bossi, S. Sortino, F. M. Raymo, J. Phys. Chem. C 2012, 116, 6058.
[56] J. Cusido, S. S. Ragab, E. R. Thapaliya, S. Swaminathan, J. Garcia-Amorós, M. J. Roberti, B. Araoz, M. M. A. Mazza, S. Yamazaki, A. M. Scott, F. M. Raymo, M. L. Bossi, J. Phys. Chem. C. 2016, 120, 12860.

[57] F. M. Raymo, M. Tomasulo, J. Phys. Chem. A 2005, 109, 7343.

[58] E. Deniz, M. Tomasulo, R. A. DeFazio, B. D. Watson, F. M. Raymo, Phys. Chem. Chem. Phys. 2010, 12, 11630.

[59] B. Seefeldt, R. Kasper, M. Beining, J. Mattay, J. Arden-Jacob, N. Kemnitzer, K. H. Drexhage, M Heilemann, M. Sauer, Photochem. Photobiol. Sci. 2010, 9, 213.

[60] I. Yildiz, S. Impellizzeri, E. Deniz, B. McCaughan, J. F. Callan, F. I. M. Raymo, $J$. Am. Chem. Soc. 2011, 133, 871.

[61] J. Whelan, D. Abdallah, K. Piskorz, J. T. C. Wojtyk, J. M. Dust, J.-M. Nunzi, S. Hoz, E. Buncel, Phys. Chem. Chem. Phys. 2012, 14 13684.

[62] G. Marriott, S. Mao, T. Sakata, J. Ran, D. K. Jackson, C. Petchprayoon, T. J. Gomez, E. Warp, O. Tulyathan, H. L. Aaron, E. Y. Isacoff, Y. Yan, Proc. Natl. Acad. Sci. USA 2008, 105, 17789.

[63] Y. Xiong, P. Rivera-Fuentes, E. Sezgin, A Vargas Jentzsch, C. Eggeling, H. L. Anderson, Org. Lett. 2016, 18, 3666.

[64] Y. Xiong, A. Vargas Jentzsch, J. Osterrieth, E. Sezgin, I. V. Sazanovich, K. Reglinski, S. Galiani, A. W. Parker, C. Eggeling, H. L. Anderson, Chem. Sci. 2018, 9, 3029.

[65] M. Irie, Chem. Rev. 2000, 100, 1685

[66] M. Herder, B. M. Schmidt, L. Grubert, M. Pätzel, J. Schwarz, S. Hecht, J. Am. Chem. Soc. 2015, 137, 2738.

[67] M. Bossi, V. Belov, S. Polyakova, S. W. Hell, Angew. Chem. Int. Ed. 2006, 45, 7462.

[68] C. Li, H. Yan, L.-X. Zhao, G.-F. Zhang, Z. Hu, Z. L. Huang, M.-Q. Zhu, Nat. Commun. 2014, 5,1 .

[69] K. Uno, H. Niikura, M. Morimoto, Y. Ishibashi, H. Miyasaka, M. Irie, J. Am. Chem. Soc 2011, 133,13558 .
[70] O. Nevskyi, D. Sysoiev, A. Oppermann, T. Huhn, D. Wöll, Angew. Chem. Int. Ed. 2016, 55 , 12698.

[71] B. Roubinet, M. L. Bossi, P. Alt, M. Leutenegger, H. Shojaei, S. Schnorrenberg, S. Nizamov, M. Irie, V. N. Belov, S. W. Hell, Angew. Chem. Int. Ed. 2016, 55, 15429.

[72] B. Roubinet, M. Weber, H. Shojaei, M. Bates, M. L. Bossi, V. N. Belov, M. Irie, S. W. Hell, J. Am. Chem. Soc. 2017, 139, 6611.

[73] C. Li, Z. Hu, M. P. Aldred, L. X. Zhao, H. Yan, G. F. Zhang, Z. L. Huang, A. D. Q. Li, M. Q. Zhu, Macromolecules 2014, 47, 8594.

[74] S. Wäldchen, J. Lehmann, T. Klein, S. Van De Linde, M. Sauer, Sci. Rep. 2015, 5, 1.

[75] C. Steinhauer, C. Forthmann, J. Vogelsagn, P. Tinnefeld, J. Am. Chem. Soc. 2008, 130, 16840.

[76] G. Lukinavičius, K. Umezawa, N. Olivier, A Honigmann, G. Yang, T. Plass, V. Mueller, L. Reymond, I. R. Corrêa Jr, Z.-G. Luo, C. Schultz, E. A. Lemke, P. Heppenstall, C. Eggeling, S. Manley, K. Johnsson, Nat. Chem. 2013, 5, 132.

[77] S. Uno, M. Kamiya, T. Yoshihara, K. Sugawara, K. Okabe, M. C. Tarhan, H. Fujita, T. Funatsu, Y. Okada, S. Tobita, Y. Urano, Nat. Chem. 2014, 6, 681 .

[78] H. Takakura, Y. Zhang, R. S. Erdmann, A. D. Thompson, Y. Lin, B. McNellis, F. RiveraMolina, S. Uno, M. Kamiya, Y. Urano, J. E. Rothman, J. Bewersdorf, A. Schepatz, D. Toomre, Nat. Biotechnol. 2017, 35, 773.

[79] S. Uno, M. Kamiya, A. Morozumi, Y. Urano, Chem. Commun. 2018, 54, 102.

[80] T. Brakemann, A. C. Stiel, G. Weber, M. Andresen, I. Testa, T. Grotjohann, M Leutenegger, U. Plessmann, H. Urlaub, C. Eggeling, M. C. Wahl, S. W. Hell, S. Jakobs, Nat. Biotechnol. 2011, 29, 942 .

[81] D. Li, L. Shao, B.-C. Chen, X. Zhang, M. Zhang, B. Moses, D. E. Milkie, J. R. Beach, J. A. Hammer, M. Pasham, T. Kirchhausen, M. A. Baird, M. W. Davidson, P. Xu, E. Betzig, Science 2015, 349, aab3500.

[82] F. Balzarotti, Y. Eilers, K. C. Gwosch, A. H. Gynnå, V. Westphal, F. D. Stefani, J. Elf, S. W. Hell, Science 2017, 355, 606. 